\title{
SWITCHING BEHAVIOR OF A SERIES CONNECTION OF A VACUUM INTERRUPTER AND A GAS CIRCUIT BREAKER
}

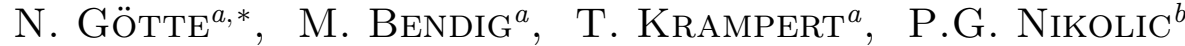 \\ ${ }^{a}$ Institute for High Voltage Technology, RWTH Aachen University, 52056 Aachen, Germany \\ ${ }^{b}$ Siemens AG, Corporate Technology, 91058 Erlangen, Germany \\ * goette@ifht.rwth-aachen.de
}

\begin{abstract}
After being in the focus of sciences' and industry's research and development activities for many years, the investigation of possible $\mathrm{SF}_{6}$ gas-alternatives has been even more intensified after the revision of the European F-Gas regulation 517/2014. As natural gases yield a significantly lower dielectric strength in comparison to $\mathrm{SF}_{6}$, new challenges arise for the design of high voltage switchgear. Vacuum interrupters are environmentally friendly, reliable and able to withstand steep rising transient recovery voltages. In the last years, first installations of switchgear based on vacuum switching technology in sub-transmission level are in operation. One option for the realization of a $\mathrm{SF}_{6}$ free high voltage switchgear for transmission level is the combination of a gas circuit breaker filled with an atmospheric gas with a vacuum interrupter in a hybrid switchgear. In this contribution the voltage distribution and switching behavior of a hybrid circuit breaker is experimentally investigated.
\end{abstract}

Keywords: vacuum circuit breaker, gas circuit breaker, hybrid circuit breaker, post-arc current, recovery voltage.

\section{Introduction}

Nowadays sulphur hexafluoride $\left(\mathrm{SF}_{6}\right)$ is the state-ofthe-art insulating and arc-quenching medium used in gas-insulated switchgear, enabling safe current interruption and high dielectric strength. At the same time, if released to the environment, $\mathrm{SF}_{6}$ is a potent greenhouse gas with a high global warming potential. After being on the agenda of research and development activities of science and industry for many years, the revision of the European regulation on fluorinated gases in 2014 even more turned the focus on the properties of possible gas-substitutes for $\mathrm{SF}_{6}$. In the field of medium-voltage technology, vacuum circuit breakers (VCB) have almost completely replaced other breaking principles. These circuit breakers are reliable, environmentally friendly and low-maintenance. In recent years, there are efforts to further develop the vacuum technology to make it suitable for the high voltage level. On one hand VCB can deal with very high rateof-rise-of-recovery voltage (RRRV) and the highest short-circuit currents, on the other hand, dielectric performance of vacuum is not rising linearly with the contact distance as it does in gases. One possible approach for an environmentally friendly switchgear for the high voltage level is a combination of VCB and gas circuit breaker (GCB) as a series connection. The interest in a hybrid circuit breaker concept has existed for almost 30 years [1], but never became a commercially available switchgear due to the success of the $\mathrm{SF}_{6}$ circuit breaker.

In this contribution interaction and switching behavior of a $\mathrm{CO}_{2}$-filled GCB and a VCB in a series connection around and after $\mathrm{CZ}$ is analyzed. It is known that the postarc current of the VCB has a great impact on voltage distribution of the series connection after CZ $[2,3]$. Therefore, this work focuses on postarc current of the VCB and the relevance for the series connection and the voltage distribution after CZ.

\section{Test Setup and Procedure}

The investigated series connection consists of a commercial VCB and a self-blast GCB model. The VCB is driven by a spring mechanism and has radial-magneticfield (RMF) contacts. The GCB model is constructed modularly so the contact distance, nozzle-system, heating volume etc. can be varied. The contact system is made of tungsten-copper ( $\mathrm{WCu}$ ) and and in this work a fixed contact distance of $40 \mathrm{~mm}$ is used. The contacts embedded into a nozzle-system with a heating channel made of polytetrafluoroethylene (PTFE), which leads through a 90 degree bend into the heating volume. The entire circuit breaker model is rotationally symmetric. $\mathrm{CO}_{2}$ is used as insulation and quenching gas. The switching arc in the GCB is initiated by a copper ignition wire with a diameter of $60 \mu \mathrm{m}$.

\subsection{Test Setup}

The used synthetic test circuit is shown in Figure 1 and consists of two parts, the high current and the high voltage circuit. After charging the capacitors $C_{\mathrm{HC}}$ and $C_{\mathrm{HV}}$ the test is initiated by the making switch (MS). A half-cycle of a sinusoidal current with a frequency of $f \approx 50 \mathrm{~Hz}$ is fed into to the deviceunder-test (DUT). Shortly before CZ the auxiliary switch (AS) is opened, the spark gap (SG) is triggered and the injection current of the high voltage 


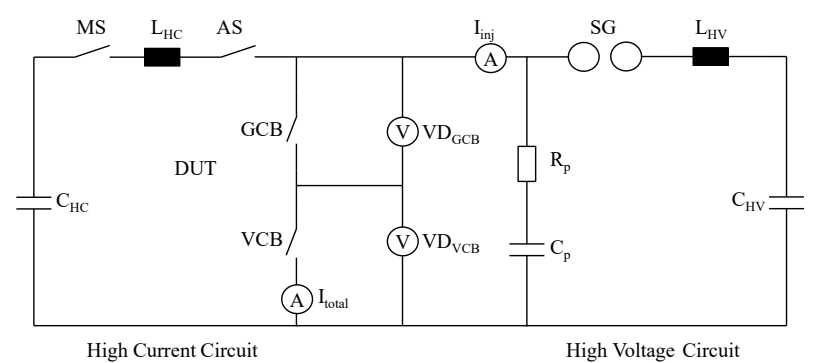

Figure 1. Test circuit consisting of high current and high voltage circuit.

circuit is superimposed onto the test current. The current steepness in $\mathrm{CZ}$ is independent of the current amplitude and is set by the charging voltage of $C_{\mathrm{HV}}$ and the resonance frequency of the high voltage circuit. The rate-of-rise-of-recovery-voltage (RRRV or $\left.\mathrm{d} u / \mathrm{d} t_{\mathrm{CZ}}\right)$ is set by the parallel network $R_{\mathrm{p}}$ and $C_{\mathrm{p}}$. The measurement data is recorded with a high resolution (12 bit), high frequency $(100 \mathrm{MHz})$ optically isolated measurement system. The postarc current is measured in the high voltage circuit $\left(I_{\mathrm{inj}}\right)$ with a coaxial shunt resistor $(25 \mathrm{~m} \Omega)$ protected by anti parallel diodes. To determine the current through the circuit breaker, the current through the stray capacitance is subtracted from the measured current value. The transient recovery voltage (TRV) over the circuit breakers are measured with two 1000:1 voltage dividers (VD), the total TRV is calculated by addition of these two signals.

\section{Experimental Results}

\subsection{Vacuum circuit breaker}

To analyze the influence of postarc current on the series connection the behavior of VCB in single operation is analyzed. Using the synthetic test circuit the current amplitude, the current steepness at $\mathrm{CZ}$ and the voltage steepness after $\mathrm{CZ}$ are set independently to determine the impact on the postarc current. With the gained information a direct comparison with the series connection is possible.

\subsubsection{Current amplitude}

The current amplitude is varied in the range from $2 \mathrm{kA}$ to $16 \mathrm{kA}$ peak current. All other parameters, especially the current steepness $(10 \mathrm{~A} / \mu \mathrm{s}), \operatorname{RRRV}(6 \mathrm{kV} / \mu \mathrm{s})$ and arcing time $(8 \mathrm{~ms})$ is set constant.

Figure 2 shows the postarc current amplitude in dependence of the current amplitude. In contrast to [4] no direct correlation with the current amplitude is visible. As only currents up to $16 \mathrm{kA}$ are investigated, the influence of contracted arc mode is supposed to be low.

\subsubsection{Current steepness and RRRV}

The variation of the current steepness, RRRV and the resulting postarc currents are shown in Figure 3.

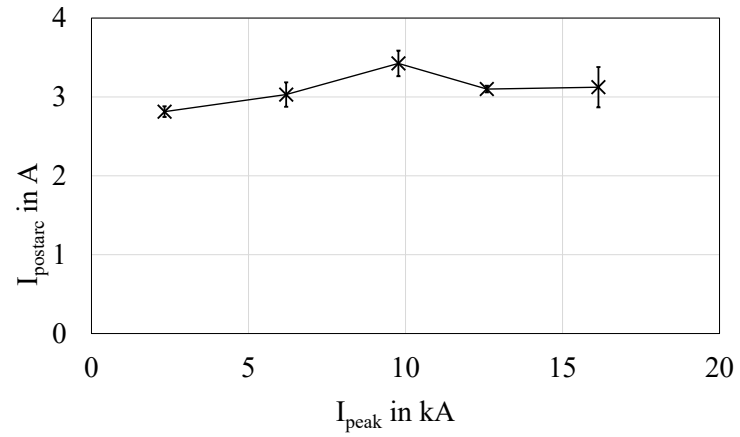

Figure 2. Variation of the current amplitude at $10 \mathrm{~A} / \mu \mathrm{s}, 6 \mathrm{kV} / \mu \mathrm{s}$ and $8 \mathrm{~ms}$ arcing time.

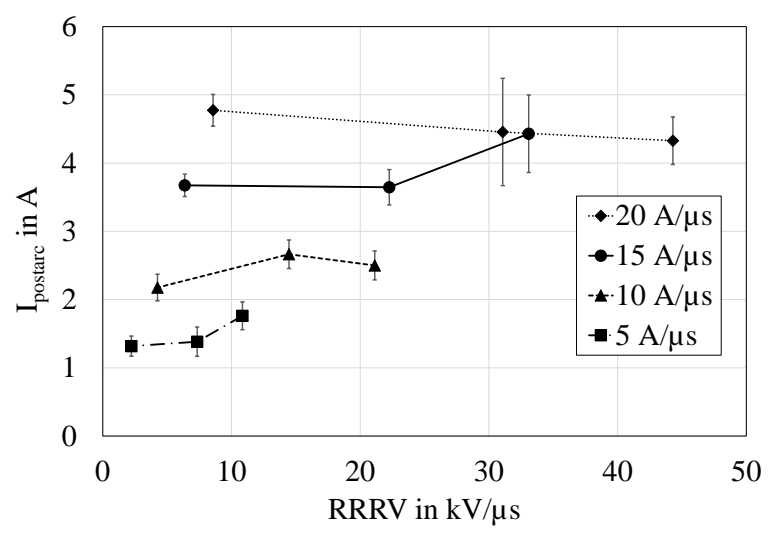

Figure 3. Variation of current steepness and $R R R V$ at $I_{\text {peak }}=2.6 \mathrm{kA}$ and $8 \mathrm{~ms}$ arcing time.

For each data point at least five measurements were carried out. To achieve different $\mathrm{d} u / \mathrm{d} t_{\mathrm{CZ}}$ values at a specific $\mathrm{d} i / \mathrm{d} t$ the parallel resistance of the parallel network $R_{\mathrm{p}}$ is varied in three steps. It is evident that the peak value of postarc current has a dependency with the set $\mathrm{d} i / \mathrm{d} t$ in $\mathrm{CZ}$. A change of $\mathrm{d} u / \mathrm{d} t_{\mathrm{CZ}}$ shows no change on the postarc current peak value of the VCB. Similar results regarding the current steepness and the postarc current in $\mathrm{CZ}$ are shown in [1].

\subsubsection{Contact distance}

From theory and other investigations it is known that the contact-distance has a direct correlation with the peak value of the postarc current [5]. The opening of the VCB is recorded using a high speed camera and synchronized with the set arcing time. In Figure 4 the measured relation between the contact distance at $\mathrm{CZ}$ and the measured maximum value of the postarc current is shown. A dependence between the maximum value of the postarc current and the contact distance is visible.

\subsection{Series Connection}

For the investigations of the series connection different types of voltage distributions after CZ are observed. The most basic type is the capacitive voltage distribution. It is observed at voltage pulses without a prior 


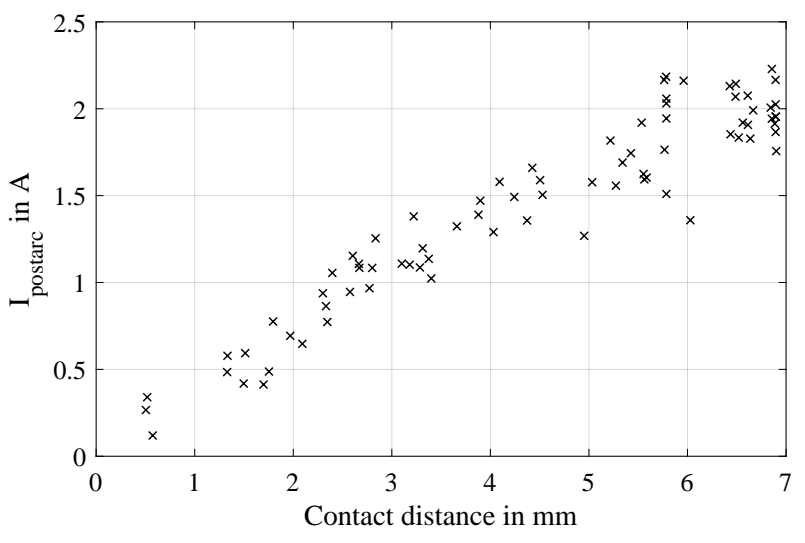

Figure 4. Variation of the contact distance of the $V C B$ at $I_{\text {peak }}=2.6 \mathrm{kA}, d i / d t=10 \mathrm{~A} / \mu \mathrm{s}$ and $\mathrm{du} / \mathrm{dt}=$ $14 \mathrm{kV} / \mu \mathrm{s}$.

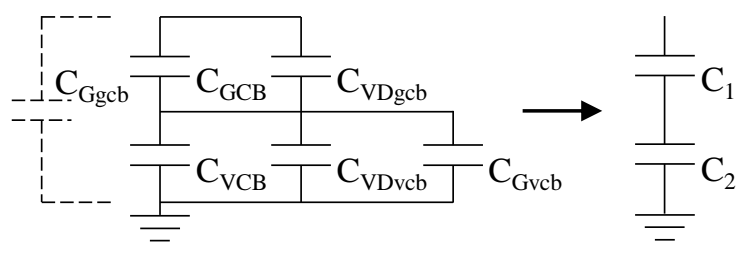

Figure 5. Relevant capacities for the voltage distribution in the test circuit.

current interruption, for example lightning impulses with open contacts.

During the interruption process in the hybrid circuit breaker, the current flows through both breakers. After CZ the differences between the postarc currents in the gas- and vacuum circuit breaker plays a major role in the voltage distribution. Typically, the postarc current of the GCB is in the range of several milliamperes, the postarc current of the VCB is in the range of several amperes. Since the current in a series connection must flow through both breakers, two scenarios are possible. The postarc current of the VCB forces a current flow through the GCB and the switching gap in the GCB stays conductive. The second possibility is that the postarc current of the VCB is suppressed by the GCB. Depended on the specific values of the postarc current a transition phase between these two operations is conceivable.

\subsubsection{Capacitive Voltage distribution}

The voltage distribution between both breakers during a switching pulse without prior current interruption depends only on the capacitive ratio. The stray capacitance of VCB is higher than that to the GCB due to the smaller contact distance and larger contact surface of the VCB. In Figure 5 the relevant capacities for the voltage distribution are given: the capacities of the breakers, the capacities of the voltage divider and the ground capacity in parallel to the circuit breaker placed on the ground side. The ground capacity in parallel to the breaker on the high voltage side (cf.
Fig. 5: $\mathrm{C}_{\mathrm{Ggcb}}$ ) has no effect on the voltage distribution. For the given results in this investigation the division ratio is about $\frac{C_{1}}{C_{2}} \approx \frac{13 \mathrm{pF}}{100 \mathrm{pF}}=0.13$. In the test setup this division ratio can easily be varied by changing the breakers on high voltage and ground side, the connection of the voltage divider or the distances to ground. Moreover, external grading capacities can be added to change the voltage distribution. To evaluate the influence of postarc current on the voltage distribution, the uninfluenced division ratio of the setup is necessary.

In Figure 6 two voltage distributions are compared. In both experiments the TRV shape is the same. In the left one the TRV is applied without a prior current interruption. Therefore the voltage is divided mainly by the capacitive division ratio and the TRV rises for the most part across the GCB. In the right part the postarc current of the VCB leads to a failure in the GCB and in the first microseconds the TRV rises across the VCB.

\subsubsection{Influence of Postarc Current}

In Figure 7 two experiments of a current interruption with the series connection are presented. In Figure 7 a) a high current- and voltage steepness is set. A postarc current of around 6 A flows through the series connection and the GCB fails. After $10-15 \mu$ s the voltage distribution changes into a capacitive voltage division. In Figure $7 \mathrm{~b}$ ) the current steepness $\mathrm{d} i / \mathrm{d} t_{\mathrm{CZ}}$ is set to $3.13 \mathrm{~A} / \mu \mathrm{s}$ and the voltage steepness at the beginning of the TRV is $0.9 \mathrm{kV} / \mu \mathrm{s}$. At this ratings the GCB is able to suppress the postarc current in the $\mathrm{VCB}$ and the TRV rises in the first $10 \mu \mathrm{s}$ exclusively over the GCB. In some experiments with these parameters, late (up to several microseconds) breakdowns of the VCB are observed. In all cases the GCB is able to withstand the whole TRV because the dielectric recovery of the $\mathrm{CO}_{2}$ gas is fast enough and no failure of the hybrid circuit breaker appeared. In single operation of the VCB no breakdown in that voltage range was recorded.

\section{Discussion}

To evaluate the operating principle of a gas and vacuum circuit breaker in a series connection the VCB is investigated separately. The postarc current of the VCB and the critical $\mathrm{d} i / \mathrm{d} t$ of the GCB at a specific switching case has the greatest influence on the voltage distribution. For the used RMF-type VCB the contact distance and the $\mathrm{d} i / \mathrm{d} t_{\mathrm{CZ}}$ changes the values of the postarc current.

When the postarc current of the VCB is suppressed by the GCB, late breakdowns of the VCB occur. It is suspected, that the suppression of the post arc current directly relates to the VCBs dielectric recovery. In theory the charged particles in the contact gap of the $\mathrm{VCB}$ are removed by the postarc current with the help of the TRV. When this process is suppressed by 

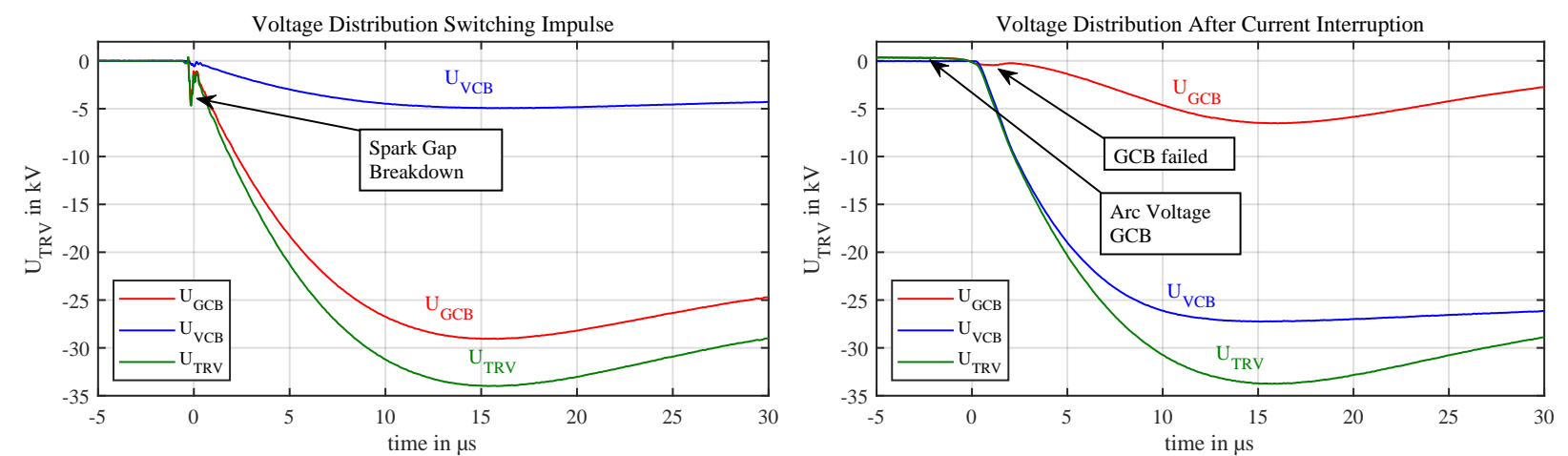

Figure 6. Comparison of voltage distribution. Left: Without current interruption. Capacitive voltage distribution. Right: Voltage distribution after current interruption. Failed interruption in the GCB due to the postarc current of the $V C B$.

a) Voltage Distribution - With Postarc Current

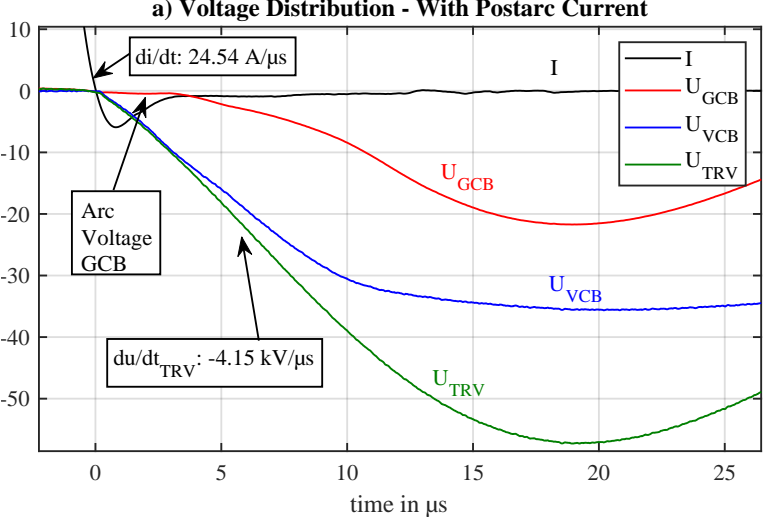

b) Voltage Distribution - No Postarc Current

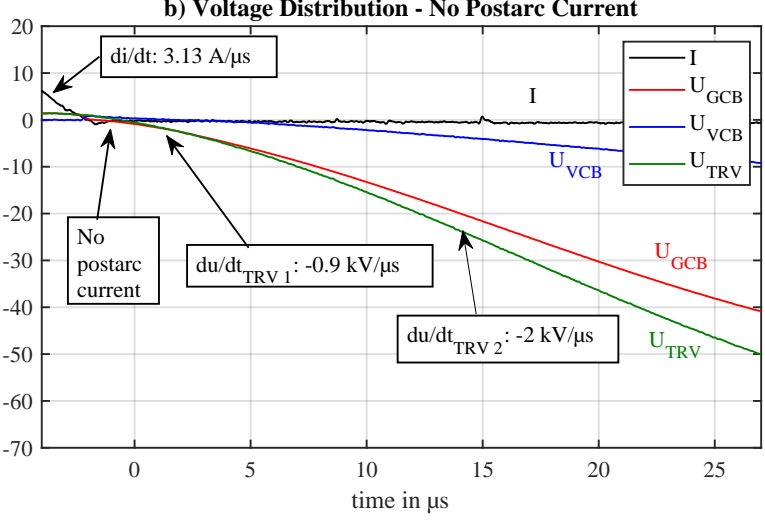

Figure \%. a) Voltage distribution and postarc. GCB failed during postarc phase. b) Voltage distribution, postarc shut off by GCB.

the GCB, the dielectric performance of the $\mathrm{VCB}$ is decreased.

\section{Conclusions}

During the interruption process of a hybrid circuit breaker, two different scenarios can be described.

1. For high current steepness in $\mathrm{CZ}$ or low interruption capability of the GCB, it is not able to interrupt the current. Consequently, the GCB stays conductive during the postarc phase of the VCB. The arc quenching takes place in the VCB and nearly the whole TRV rises across its contacts. After the postarc phase, in the transition phase, the distribution ratio changes with the speed of dielectric recovery of the GCB. In the last phase the voltage distribution depends only on the capacitive ratio.

2. For low current steepness in $\mathrm{CZ}$ or high interruption performance of the GCB, the postarc current of the VCB is suppressed by the GCB. The arc quenching takes place in the GCB. The charged particles between the VCB contacts can only be discharged very slowly and the TRV rises almost exclusively across the GCB. Late breakdowns appear in the VCB.

For the voltage distribution and the interruption process of a hybrid circuit breaker, the postarc current of the VCB plays a major role. The probability of a failure of the GCB and therefore a rise of the TRV in the first microseconds above the VCB increases with the peak value of the postarc current.

\section{References}

[1] K. Natsui et al. Voltage distribution characteristics of series connected $\mathrm{SF}_{6}$ gas and vacuum interrupters immediately after large AC current interruption. IEEE Transactions on Power Delivery, 3(1):241-248, 1988. doi:10.1109/61.4251.

[2] R. P. P. Smeets, V. Kertesz, D. Dufournet, D. Penache, and M. Schlaug. Interaction of a vacuum arc with an $\mathrm{SF}_{6}$ arc in a hybrid circuit breaker during high-current interruption. IEEE Transactions on Plasma Science, 35(4):933-938, 2007. doi:10.1109/TPS.2007.896919.

[3] X. Cheng et al. Dynamic dielectric recovery synergy of hybrid circuit breaker with $\mathrm{CO}_{2}$ gas and vacuum interrupters in series. IEEE Transactions on Plasma Science, 45(10):2885-2892, 2017. doi:10.1109/TPS.2017.2743691.

[4] N. Ide, O. Tanaka, S. Yanabu, S. Kaneko, S. Okabe, and Y. Matsui. Interruption characteristics of doublebreak vacuum circuit breakers. IEEE Transactions on Dielectrics and Electrical Insulation, 15(4):1065-1072, 2008. doi:10.1109/TDEI. 2008.4591229.

[5] K. Steinke, M. Lindmayer, and K.-D. Weltmann. Post-arc currents of vacuum interrupters with axial magnetic field contacts under high current and voltage stress. In Proceedings ISDEIV, pages 475-480, 2000. doi:10.1109/DEIV.2000.879030. 